\title{
Proposed measurement of tagged deep inelastic scattering in Hall A of Jefferson lab
}

Cite as: AIP Conference Proceedings 1819, 030004 (2017); https://doi.org/10.1063/1.4977122 Published Online: 03 March 2017

R. A. Montgomery, J. R. M. Annand, D. Dutta, C. E. Keppel, P. King, B. Wojtsekhowski, J. Zhang, the TDIS Collaboration, and the SBS Collaboration

ARTICLES YOU MAY BE INTERESTED IN

Group Photo: Diffraction 2016, International Workshop on Diffraction in High-Energy Physics AIP Conference Proceedings 1819, 010003 (2017); https://doi.org/10.1063/1.4977115

Impact factor for exclusive diffractive dijet production with NLO accuracy AIP Conference Proceedings 1819, 030009 (2017); https://doi.org/10.1063/1.4977127

On the color dipole picture

AIP Conference Proceedings 1819, 030007 (2017); https://doi.org/10.1063/1.4977125

Challenge us.

What are your needs for periodic signal detection?

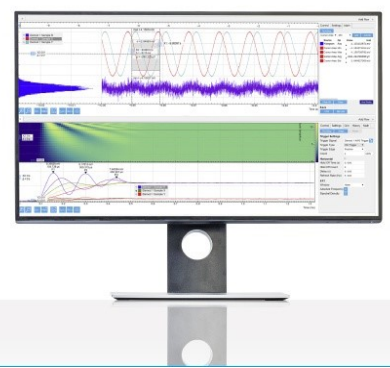

View Online

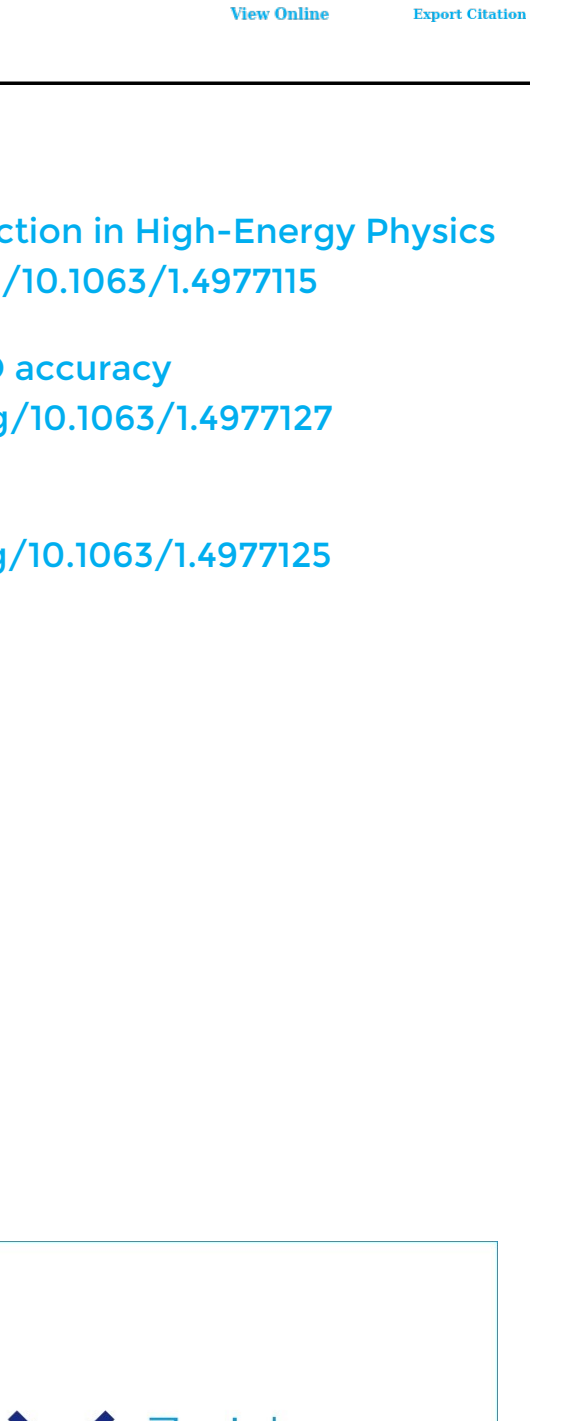

Zurich Instruments 


\title{
Proposed Measurement of Tagged Deep Inelastic Scattering in Hall A of Jefferson Lab
}

\author{
R.A. Montgomery ${ }^{1, \text { a) }}$, J.R.M. Annand ${ }^{1}$, D. Dutta ${ }^{2}$, C.E. Keppel ${ }^{3}$, P. King ${ }^{4}$, B. \\ Wojtsekhowski ${ }^{3}$, J. Zhang ${ }^{5}$, the TDIS Collaboration and the SBS Collaboration \\ ${ }^{1}$ University of Glasgow, Glasgow G12 8QQ, Scotland, UK \\ ${ }^{2}$ Mississippi State University, Mississippi State, MS 39762, USA \\ ${ }^{3}$ Thomas Jefferson National Accelerator Facility, Newport News, VA 23606, USA \\ ${ }^{4}$ Ohio University, Athens, OH 45701, USA \\ ${ }^{5}$ University of Virginia, Charlottesville, VA 22904, USA

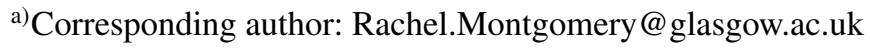

\begin{abstract}
A tagged deep inelastic scattering (TDIS) experiment is planned for Hall A of Jefferson Lab, which will probe the mesonic content of the nucleon directly. Low momentum recoiling (and spectator) protons will be measured in coincidence with electrons scattered in a deep inelastic regime from hydrogen (and deuterium) targets, covering kinematics of $8<W^{2}<18 \mathrm{GeV}^{2}$, $1<Q^{2}<3(\mathrm{GeV} / c)^{2}$ and $0.05<x<0.2$. The tagging technique will help identify scattering from partons in the meson cloud and provide access to the pion structure function via the Sullivan process. The experiment will yield the first TDIS results in the valence regime, for both proton and neutron targets. We present here an overview of the experiment.
\end{abstract}

\section{INTRODUCTION}

Following the recent $12 \mathrm{GeV}$ upgrade at Jefferson Lab [1], a pioneering TDIS experiment [2] has been proposed and is planned to run in experimental Hall A. The experiment aims to probe the nucleon's mesonic content directly, and facilitate extraction of the pion Structure Function (SF) via the Sullivan process [3]. This process can be studied by measuring the contribution to electron Deep Inelastic Scattering (DIS) off the meson cloud of a nucleon target at rest.

The pion content of the nucleon, in particular, is considered fundamental to our knowledge of nucleon structure and interactions (a review is given in [4]). For instance, study of the pion allows further understanding of both the simplest QCD state and the Goldstone boson of QCD. The pion is also considered to play a key role in the nucleon's long-range structure, and could help understand the origin of the $\bar{d} / \bar{u}$ flavour asymmetry of the nucleon sea.

Measurements of the pion's partonic stucture, however, are limited. Drell-Yan (DY) measurements provide pion SF data in the valence regime $[5,6,7]$. In the $q \bar{q}$-sea region, at low Bjorken- $x(x)$ and high $Q^{2}$, the pion SF was extracted from hard diffractive scattering at HERA, where leading neutrons were tagged in ep $\rightarrow$ eXN $[8,9]$. The TDIS experiment will yield the first direct measurements of tagged proton and neutron DIS in the valence region, providing access to both neutral and charged pion clouds. Comparing with HERA results, TDIS measurements will allow studies of evolution between different kinematic regimes. Comparison with DY results will provide independent cross-checks of various conflicting theoretical models in the valence regime, and extend the measurements to neutral pions.

\section{TAGGED DEEP INELASTIC SCATTERING}

The TDIS investigations will measure the semi-inclusive reactions $H\left(e, e^{\prime} p\right) X$ and $D\left(e, e^{\prime} p p\right) X$ in the target fragmentation region. A tagging technique and the selection of specific kinematics will help identify events where the scattering took place from the meson cloud. For the hydrogen (H) target, DIS events from the neutral pion in proton-pion fluctuations will be tagged, measuring the recoiling proton in coincidence with the scattered electron. For deuterium (D), 
electrons scattered from charged pions arising from neutron-pion fluctuations occurring during neutron charge exchange processes will be selected. Both the outgoing recoil and backwards-going spectator protons will be measured in vertex coincidence with the electron. This technique is similar to that used by the BONUS experiment, to enhance scattering from the neutron in the deuteron $[11,12]$. The four-momentum transfer squared $t \equiv k^{2}=\left(p-p^{\prime}\right)^{2}$, where $p$ and $p^{\prime}$ are the initial and final four-momenta of the nucleon, must be low $\left(|t|<0.2(\mathrm{GeV} / c)^{2}\right)$, to allow extrapolation towards the pion mass pole. This corresponds to measured protons of very low momenta, from $60-400 \mathrm{MeV} / c$. The pion and recoiling hadronic system in both target cases are undetected but reconstructed.

Several phenomenological models of the meson cloud and its contribution to the nucleon SF exist $[9,13,14,15]$. The framework used to calculate pion and tagged semi-inclusive SF's for TDIS was based on the phenomenological pion cloud model developed by Hobbs et al. [13, 14, 15]. Full description of the theory is found in [2, 13]. The TDIS measurements will ultimately extract the tagged $\mathrm{SF} F_{2}^{T}\left(x, Q^{2}, z, t\right)$, i.e. the semi-inclusive $\mathrm{SF}$ of the recoil proton. This is achieved through application of a ratio method to the data, resulting in the cancellation of various systematic uncertainties. The measured ratio, $R_{T}$, of tagged to un-tagged DIS cross-sections is given in Equation (1):

$$
R^{T}=\frac{\frac{d^{4} \sigma\left(e p \rightarrow e^{\prime} X p^{\prime}\right)}{d x d Q^{2} d z d t}}{\frac{d^{2} \sigma\left(e p \rightarrow e^{\prime} X\right)}{d x d Q^{2}}} \Delta z \Delta t \sim \frac{F_{2}^{T}\left(x, Q^{2}, z, t\right)}{F_{2}^{p}\left(x, Q^{2}\right)} \Delta z \Delta t .
$$

The quantity $z=\frac{k^{+}}{p^{+}}$is the light-cone momentum fraction of the initial nucleon carried by the interacting pion. The un-tagged proton $\mathrm{SF} \mathrm{F}_{2}^{p}\left(x, Q^{2}\right)$ will be measured simultaneously and is also well-known from global data. The tagged $\mathrm{SF} F_{2}^{T}\left(x, Q^{2}, z, t\right)$ is therefore obtained as shown in Equation (2),

$$
F_{2}^{T}\left(x, Q^{2}, z, t\right)=\frac{R^{T}}{\Delta z \Delta t} F_{2}^{p}\left(x, Q^{2}\right) .
$$

An analogous method may be applied for the neutron target. The measurement will span a broad range of $t$ and $x$, and the pion SF $F_{2}^{\pi}$ may then be extracted using various models of the pion cloud $[2,9,13]$. Since the extraction is model dependent, the effect on $F_{2}^{\pi}$ of different models, e.g. phenomenological, DSE-inspired or Regge models, will be investigated. The pion SF extraction will also require several corrections, for example due to non-pion pole contributions, $\Delta$ and $N^{*}$ resonances, absorptive effects and pion flux uncertainties. These corrections are minimised by tagging the recoiling proton momentum as low as possible. In spite of the above complications, the shape of the pion SF at large $x$ could be obtained reliably from these data, which would be essential in view of the current tensions between the DY data and DSE predictions.

\section{EXPERIMENTAL SET UP}

A schematic of the proposed experimental layout is shown in Figure 1. An electron beam, of $11 \mathrm{GeV}$ energy, $50 \mu \mathrm{A}$ current and $3 \times 10^{36} \mathrm{~Hz} / \mathrm{cm}^{2}$ luminosity, is incident upon a thin-walled straw target. The target has a radius of $5 \mathrm{~mm}$ and a length of $400 \mathrm{~mm}$. The target is filled with either $\mathrm{H}$ or D gas, maintained at $77 \mathrm{~K}$ and $1 \mathrm{~atm}$. This is surrounded by a Radial Time Projection Chamber (RTPC) to measure the protons, which is described in further detail below. Both the target and RTPC are contained within a solenoid to allow for momentum analysis of the protons' tracks and to reduce electromagnetic background. The solenoid has a maximum field of $4.7 \mathrm{~T}$ at its centre. The scattered electron will be detected by the Super BigBite Spectrometer (SBS), which is currently under construction. It is an open-geometry and configurable spectrometer, comprised of a dipole magnet and several modular detectors. For the TDIS set-up, the SBS will be $2 \mathrm{~m}$ from the target at a scattering angle of $12^{\circ}$, providing a solid angle of $\sim 50 \mathrm{msr}$. It will be configured for electron detection, incorporating five large-area gaseous electron multiplier (GEM) chambers [17], a threshold Cherenkov detector (Gas Cher.) and a segmented calorimeter (large angle calorimeter, LAC). The Cherenkov detector and LAC will be combined to provide the electron identification and trigger.

\section{Radial Time Projection Chamber}

The RTPC is conceptually similar to that previously used for the BONUS and EG6 experiments at CLAS [16]. It will allow for track reconstruction of the low-momentum protons with high resolution, and $d E / d x$ analysis for successful proton identification. It has an outer radius $(r)$ of $150 \mathrm{~mm}$ and it is assumed that it will be filled with a $90: 10$ helium : methane $\left(\mathrm{He}: \mathrm{CH}_{4}\right)$ gas mix, maintained at $77 \mathrm{~K}$ and $0.2 \mathrm{~atm}$. A ring of field wires divide the He volume into an 


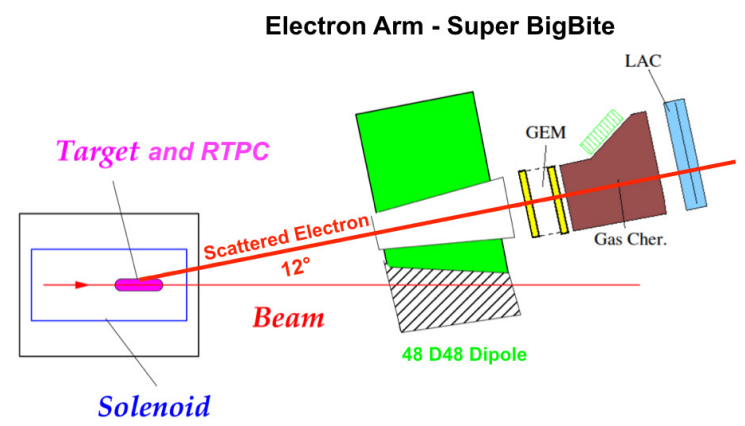

FIGURE 1. Experimental set up for the proposed TDIS experiment in Hall A of Jefferson Lab.

insensitive region at $r<50 \mathrm{~mm}$ and a sensitive region at $50<r<150 \mathrm{~mm}$. Ionisation produced at $50<r<150 \mathrm{~mm}$ is swept by a radial electric field, of average value $\sim 500 \mathrm{~V} / \mathrm{cm}$, to the curved triple-layer GEM detectors at $r=150 \mathrm{~mm}$.

Beam related background processes have been simulated using Geant 4 [10]. The simulated geometry is shown in Figure 2 (a), with: solenoidal field in yellow; beam pipe in grey; RTPC in white; outer GEM layer in red. Example proton tracks within the geometry are shown in Figure 2 (b). These simulations demonstrated that Møller electrons and other electromagnetic background in the target vicinity will be contained by the solenoidal field. Protons produced by photo-nuclear processes will represent the most challenging background. For the $\mathrm{H}$ target this will mostly be rejected after elastic scattering cuts, accepting only protons with scattering angles of $30<\theta<70^{\circ}$ for example. For $\mathrm{D}$, quasifree scattering and quasi-deuteron processes will cause the majority of background protons, with kinematics similar to TDIS protons. For $\mathrm{H}$ and $\mathrm{D}$, background proton rates of around $\mathrm{MHz}$ and $10^{2} \mathrm{MHz}$ respectively are expected in kinematics similar to TDIS protons. True TDIS protons will be separated from accidentals through coincidence time and position vertexing, using information from both the SBS and RTPC.

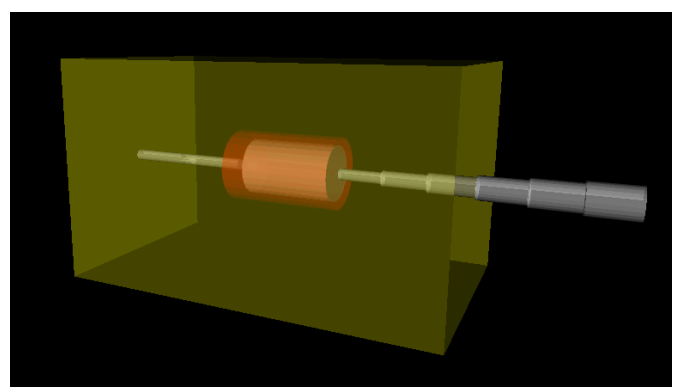

(a)

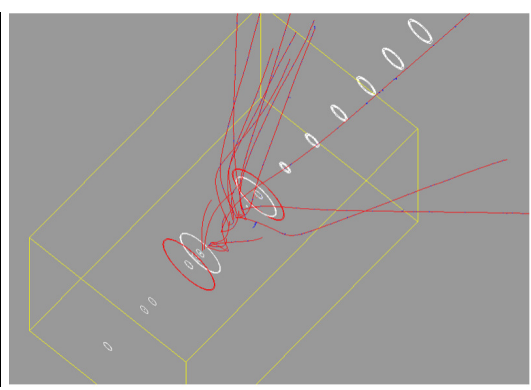

(b)

FIGURE 2. (a) RTPC geometry described in Geant4, and (b) simulated proton tracks with momentum $250 \mathrm{MeV} / c$ in geometry.

The most challenging issue concerning background rates will be the occupancy of the read-out pads of the GEM chambers, due to rather long ionisation drift times within the RTPC. An interface has been developed which combines the Geant 4 simulation with Garfield++ software [18]. Geant4 is used to track protons and their energy deposit through the geometry, until the track enters the sensitive He region where the track is killed and its kinematical information is passed to Garfield++. Garfield++ is then used to simulate ionisation created by the proton within the He sensitive region and the subsequent drift of electrons under the influence of the RTPC electromagnetic field. Preliminary simulations indicate electron drift times ranging up to values on the order of $\sim 20 \mu \mathrm{s}$, although the electron drift times may be further fine-tuned if required through optimisation of the gas mixture settings.

\section{PROJECTED RESULTS}

Projected SF results are given in Figure 3. Figure 3 (a) shows $\mathrm{H}$ target results, at a central recoil proton momentum bin $\Delta k=200-250 \mathrm{MeV} / c$ and a recoil proton scattering angle range $\Delta \theta_{p}$ of $30-70^{\circ}$. The SF's shown are: full inclu- 


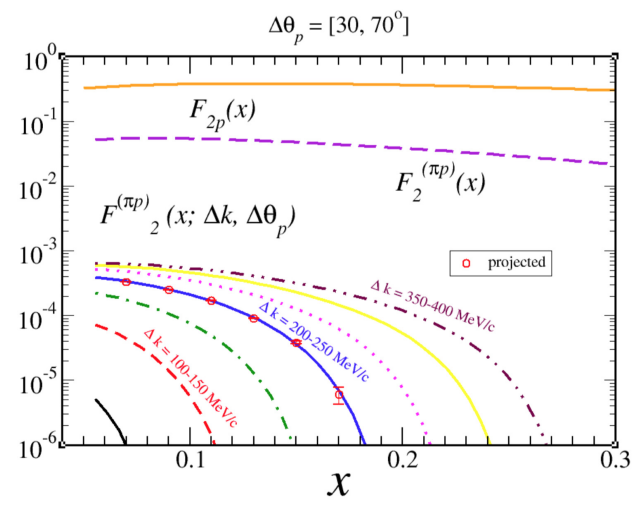

(a)

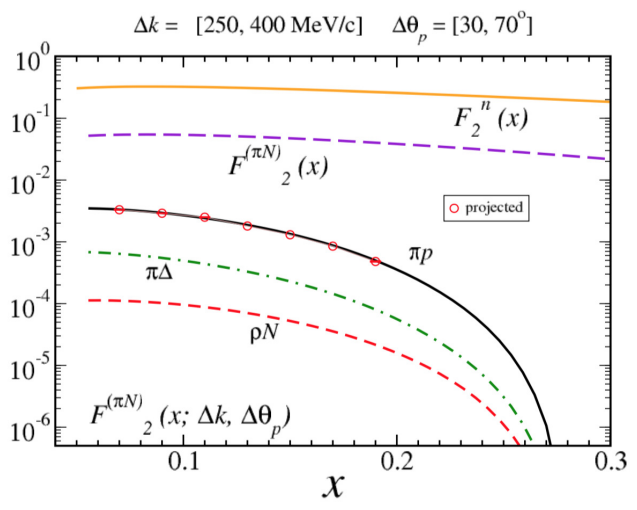

(b)

FIGURE 3. Projected structure function measurements in example bins for (a) hydrogen and (b) deuterium targets [2].

sive proton $F_{2}^{p}(x)$ (orange); full pionic content of the proton $F_{2}^{(\pi p)}(x)$ (purple); tagged semi-inclusive $F_{2}^{(\pi p)}\left(x ; \Delta k, \Delta \theta_{p}\right)$ projections for differing $\Delta k$ bins (coloured lines). The relative $F_{2}^{(\pi p)}\left(x ; \Delta k, \Delta \theta_{p}\right)$ strengths compared with $F_{2}^{p}(x)$ demonstrate why a high beam luminosity is required. Figure 3 (b) shows analogous SF's for the D target and projections for the tagged semi-inclusive out-going recoil proton SF ( $\pi p$, black), for a larger recoil momentum range and the same $\Delta \theta_{p}$ bin. Comparisons in this case are given with background processes which contribute to the signal. The $\rho^{-}$component $\left(\rho N\right.$, red) is tiny in magnitude compared to the $\pi^{-} p$ channel, and the $\pi^{0} \Delta^{0}+\pi^{-} \Delta^{+}$channel contribution $(\pi \Delta$, green) may be further reduced by kinematic cuts. For supplementary projected results and more details see [2].

\section{SUMMARY}

The TDIS experiment proposed for Hall A in Jefferson lab will directly probe the meson cloud of the nucleon via the Sullivan process, and provide the first direct measurements of tagged proton and neutron SF's in the valence regime. The experimental technique will also provide a gateway for future similar tagging approaches aiming to study the nucleon's mesonic component, for example at Jefferson Lab or at proposed colliders such as the EIC.

\section{REFERENCES}

[1] A. Lung, https://www.jlab.org/12GeV/, August 25, 2016.

[2] C.E. Keppel et al., Measurement of Tagged Deep Inelastic Scattering (TDIS), A Hall-A and SBS Collaboration Proposal, Jefferson Lab Experiment PR12-15-006, May 15, 2015.

[3] J.D. Sullivan, Phys. Rev. D 5, 1732 (1972).

[4] R. Holt and C. Roberts, Rev. of Mod. Phy. 82, 2991 (2010).

[5] J. S. Conway et al., Phys. Rev. D 39, 39 (1989).

[6] B. Betevet al., Z. Phys. C 28, 9 (1985).

[7] P. J. Sutton, A. D. Martin, W. J. Stirling and R. G. Roberts, Phys. Rev. D 45, 2349 (1992).

[8] The H1 Collaboration, F. D. Aaron, C. Alexa et al., Eur. Phys. J. C 68, 381 (2010).

[9] J. R. McKenney, Nobuo Sato, W. Melnitchouk and Chueng-Ryong Ji, Phys. Rev. D 93, 054011 (2016).

[10] J. Allison et al., Nucl. Ins. and Meth. in Phys. Research A 506, 250 (2003).

[11] N. Baillie et al., Phys. Rev. Lett. 108, 199902 (2012).

[12] S. Tkachenko, Phys. Rev. C 89, 045206 (2014).

[13] T. J. Hobbs, Few-Body Syst 56, 363 (2015).

[14] H. Holtmann, A. Szczurek and J. Speth, Nucl. Phys. A 596, 631 (1996).

[15] W. Melnitchouk and A. W. Thomas, Z. Phys. A 353, 311 (1995).

[16] H. Fenker et al., Nucl. Ins. and Meth. in Phys. Research A 592, 273 (2008).

[17] K. Gnanvo et al., Nucl. Ins. and Meth. in Phys. Research A 782, 77 (2015).

[18] CERN, http://garfieldpp.web.cern.ch/garfieldpp/, September 17, 2015. 